\title{
Elevated Urine Alkaline Phosphatase
}

National Cancer Institute

\section{Source}

National Cancer Institute. Elevated Urine Alkaline Phosphatase. NCI Thesaurus. Code C122821.

An elevated concentration of alkaline phosphatase in the urine. 